\title{
EXISTENCE RESULTS FOR GENERAL INEQUALITY PROBLEMS WITH CONSTRAINTS
}

\author{
GEORGE DINCĂ, PETRU JEBELEAN, AND DUMITRU MOTREANU
}

Received 25 February 2002

\section{To Professor Jean Mawhin on occasion of his 60th birthday}

This paper is concerned with existence results for inequality problems of type $F^{0}(u ; v)+\Psi^{\prime}(u ; v) \geq 0$, for all $v \in X$, where $X$ is a Banach space, $F: X \rightarrow \mathbb{R}$ is locally Lipschitz, and $\Psi: X \rightarrow(-\infty+\infty)$ is proper, convex, and lower semicontinuous. Here $F^{0}$ stands for the generalized directional derivative of $F$ and $\Psi^{\prime}$ denotes the directional derivative of $\Psi$. The applications we consider focus on the variational-hemivariational inequalities involving the $p$-Laplacian operator.

\section{Introduction}

The paper deals with nonlinear inequality problems of type

$$
F^{0}(u ; v-u)+h(v)-h(u) \geq 0, \quad \forall v \in C,
$$

where $F^{0}$ stands for the generalized directional derivative of a locally Lipschitz functional $F$ (in the sense of Clarke [5]), $h$ is a convex, lower semicontinuous (in short, l.s.c.), and proper function, and $C$ is a nonempty, closed, and convex subset of a Banach space $X$. It is clear that in problem (1.1) we can put $h+I_{C}$ in place of $h$, where $I_{C}$ denotes the indicator function of the set $C$, to give the formulation with $v$ arbitrary in $X$. However, we keep the statement (1.1) for allowing various possible choices separately on the data $h$ and $C$.

The type of problem stated in (1.1) fits in the framework of the nonsmooth critical point theory developed by Motreanu and Panagiotopoulos [9], which is constructed for the nonsmooth functionals having the form

$$
\Phi=\Psi+F
$$

with $\Psi$ convex, l.s.c., and proper, and $F$ locally Lipschitz. Namely, a solution of 
(1.1) means, in fact, a critical point of the associated nonsmooth functional (1.2) with $\Psi=h+I_{C}$.

The existence results in the present paper extend different theorems in the smooth and nonsmooth variational analyses (see, for comparison, Ambrosetti and Rabinowitz [2], Chang [4], Dincă et al. [8], Motreanu and Panagiotopoulos [9], Rabinowitz [10], and Szulkin [11]). In this respect, we solve problems of type

$$
F^{0}(u ; v)+\Psi^{\prime}(u ; v) \geq 0, \quad \forall v \in X
$$

where $\Psi^{\prime}$ stands for the directional derivative of a convex, proper, 1.s.c. functional $\Psi$. Consequently, we are able to handle the abstract hemivariational inequality problem

$$
F^{0}(u ; v-u)+\langle d \varphi(u), v-u\rangle \geq 0, \quad \forall v \in C,
$$

where $\varphi$ is a convex, Gâteaux differentiable functional and $d \varphi$ is its differential. In particular, this contains the differential inclusion problem

$$
d \varphi(u) \in \partial(-F)(u)
$$

which we considered in our previous paper [8].

The rest of the paper is organized as follows. In Section 2, we briefly recall several elements of nonsmooth critical point theory developed by Motreanu and Panagiotopoulos [9]. In Section 3, we study some general inequality problems in relation with the nonsmooth critical point theory. Section 4 presents applications for different discontinuous boundary value problems with $p$-Laplacian.

\section{Notions and preliminary results}

Let $X$ be a real Banach space and $X^{*}$ its dual. The generalized directional derivative of a locally Lipschitz function $F: X \rightarrow \mathbb{R}$ at $u \in X$ in the direction $v \in X$ is defined by

$$
F^{0}(u ; v)=\limsup _{w \rightarrow u, t \succ 0} \frac{F(w+t v)-F(w)}{t} .
$$

The generalized gradient (in the sense of Clarke [5]) of $F$ at $u \in X$ is defined to be the subset of $X^{*}$ given by

$$
\partial F(u)=\left\{\eta \in X^{*}: F^{0}(u ; v) \geq\langle\eta, v\rangle, \forall v \in X\right\},
$$

where $\langle\cdot, \cdot\rangle$ stands for the duality pairing between $X^{*}$ and $X$.

Let $\Psi: X \rightarrow(-\infty,+\infty]$ be a proper (i.e., $D(\Psi):=\{u \in X: \Psi(u)<+\infty\} \neq \varnothing$ ), convex, and 1.s.c. function and let $F: X \rightarrow \mathbb{R}$ be locally Lipschitz.

We define the functional $\Phi: X \rightarrow(-\infty,+\infty]$ by $\Phi=\Psi+F$. 
Definition 2.1 Motreanu and Panagiotopoulos [9]. An element $u \in X$ is called critical point of the functional $\Phi$ if this inequality holds

$$
F^{0}(u ; v-u)+\Psi(v)-\Psi(u) \geq 0, \quad \forall v \in X
$$

Definition 2.2 Motreanu and Panagiotopoulos [9]. The functional $\Phi$ is said to satisfy the Palais-Smale condition if every sequence $\left\{u_{n}\right\} \subset X$ for which $\Phi\left(u_{n}\right)$ is bounded and

$$
F^{0}\left(u_{n} ; v-u_{n}\right)+\Psi(v)-\Psi\left(u_{n}\right) \geq-\varepsilon_{n}\left\|v-u_{n}\right\|, \quad \forall v \in X
$$

for a sequence $\left\{\varepsilon_{n}\right\} \subset \mathbb{R}^{+}$with $\varepsilon_{n} \rightarrow 0$, contains a strongly convergent subsequence in $X$.

For the proof of the next theorem, we refer the reader to [8, Proposition 2.1] and [9, Corollary 3.2] (also see [8, Theorem 2.2]).

Theorem 2.3. (i) If $u \in X$ is a local minimum for $\Phi$, then $u$ is a critical point of $\Phi$.

(ii) If $\Phi$ satisfies the Palais-Smale condition and there exist a number $\rho>0$ and a point $e \in X$ with $\|e\|>\rho$ such that

$$
\inf _{\|v\|=\rho} \Phi(v)>\Phi(0) \geq \Phi(e)
$$

then $\Phi$ has a nontrivial critical point.

Remark 2.4. Definitions 2.1 and 2.2 recover and unify the nonsmooth critical point theories (and a fortiori the smooth critical point theory, see, e.g., Ambrosetti and Rabinowitz [2] and Rabinowitz [10]) due to Chang [4] and Szulkin [11]. Precisely, if $\Psi=0$, Definitions 2.1 and 2.2 reduce to the corresponding definitions of Chang [4], while if $F \in C^{1}(X, \mathbb{R})$, then Definitions 2.1 and 2.2 coincide with those in Szulkin [11].

\section{Critical points as solutions of inequality problems}

Throughout this section, $\left(X,\|\cdot\|_{X}\right)$ is a real reflexive Banach space, compactly embedded in the real Banach space $\left(Z,\|\cdot\|_{Z}\right)$. Let $\mathscr{F}: Z \rightarrow \mathbb{R}$ be a locally Lipschitz function and let $\Psi: X \rightarrow(-\infty,+\infty]$ be convex, l.s.c., and proper.

We consider the inequality problem:

$$
\text { Find } u \in D(\Psi) \text { such that }\left(\left.\mathscr{F}\right|_{X}\right)^{0}(u ; v)+\Psi^{\prime}(u ; v) \geq 0, \quad \forall v \in X \text {, }
$$

where $\left(\left.\mathscr{F}_{X}\right|_{X}\right)^{0}$ denotes the generalized directional derivative of the restriction $\left.\mathscr{F}\right|_{X}$ while $\Psi^{\prime}(u ; v)$ is the directional derivative of the convex function $\Psi$ at $u$ in the direction $v$ (which is known to exist). Note that if the Gâteaux differential $d \Psi(u)$ of $\Psi$ at $u \in D(\Psi)$ exists, then $\langle d \Psi(u), v\rangle=\Psi^{\prime}(u ; v)$, for all $v \in X$. 
604 Existence results for inequality problems

Proposition 3.1. Each solution of problem (3.1) solves the problem:

$$
\text { Find } u \in D(\Psi) \text { such that } \mathscr{F}^{0}(u ; v)+\Psi^{\prime}(u ; v) \geq 0, \quad \forall v \in X \text {. }
$$

If, in addition to our assumptions, $X$ is densely embedded in $Z$, then problems (3.1) and (3.2) are equivalent.

Proof. For $u, v \in X$, the inequality below holds

$$
\left(\left.\mathscr{F}\right|_{X}\right)^{0}(u ; v) \leq \mathscr{F}^{0}(u ; v) .
$$

This becomes an equality if $X$ is continuously and densely embedded in $Z$ (see [5, pages 46-47] and [9, pages 10-12]).

Our approach for studying problem (3.1) is variational and relies on the use of the functional

$$
\Phi=\Psi+\left.\mathscr{F}\right|_{X}: X \longrightarrow(-\infty,+\infty]
$$

which is clearly of the form required in the previous section with $F=\left.\mathscr{F}\right|_{X}$.

The next result points out the relationship between the critical points of the functional $\Phi$ in (3.4) and the solutions of problem (3.1).

Proposition 3.2. (i) If $u \in X$ is a critical point of the functional $\Phi$ in (3.4), that is,

$$
\left(\left.\mathscr{F}\right|_{X}\right)^{0}(u ; v-u)+\Psi(v)-\Psi(u) \geq 0, \quad \forall v \in X
$$

then $u$ is a solution of problem (3.1).

(ii) Conversely, assume that $u \in X$ is a solution of problem (3.1). If either $\Psi$ is Gâteaux differentiable at $u$ or $\Psi$ is continuous at $u$, then $u$ is a critical point of $\Phi$, that is, relation (3.5) holds.

Proof. (i) As $\Psi$ is proper, (3.5) obviously implies that $u \in D(\Psi)$. For an arbitrary $w \in X$, we set $v=u+t w, t>0$, in (3.5). Dividing by $t$ and then letting $t \rightarrow 0^{+}$, we arrive at the conclusion that $u$ solves problem (3.1).

(ii) Let $u \in D(\Psi)$ be a solution of problem (3.1). If $\Psi$ is Gâteaux differentiable at $u$, then

$$
\Psi(v)-\Psi(u) \geq\langle d \Psi(u), v-u\rangle=\Psi^{\prime}(u ; v-u), \quad \forall v \in X
$$

which leads to (3.5).

If $\Psi$ is continuous at $u$, then a standard result of convex analysis (see Barbu and Precupanu [3, page 106]) allows to write

$$
\Psi^{\prime}(u ; v)=\max \left\{\left\langle x^{*}, v\right\rangle: x^{*} \in \partial \Psi(u)\right\}, \quad \forall v \in X .
$$

Using the definition of the subdifferential $\partial \Psi(u)$, we obtain (3.5). 
Remark 3.3. In view of Proposition 3.2(i), each result stating the existence of critical points for $\Phi$ in (3.4) asserts a fortiori existence of solutions to problem (3.1).

Theorem 3.4. If $\Phi$ is coercive on $X$, that is,

$$
\Phi(u) \longrightarrow+\infty \quad \text { as }\|u\|_{X} \longrightarrow+\infty,
$$

then $\Phi$ has a critical point.

Proof. The compact embedding of $X$ into $Z$ implies that $\left.\mathscr{F}\right|_{X}$ is weakly continuous. We infer that $\Phi$ is sequentially weakly l.s.c. on $X$. Then, by standard theory, $\Phi$ is bounded from below and attains its infimum at some $u \in X$. From Theorem 2.3(i), $u$ is a critical point of $\Phi$.

Towards the application of Theorem 2.3(ii) to the functional $\Phi$, we have to know when $\Phi$ satisfies the Palais-Smale condition. The following lemma provides a useful sufficient condition that improves the usual results based on the celebrated hypothesis $\left(p_{5}\right)$ in [2] or $\left(p_{4}\right)$ in [10].

Lemma 3.5. Assume, in addition, that $\Psi$ and $\mathscr{F}$, entering the expression of $\Phi$ in (3.4), satisfy the following hypotheses:

(H1) $D(\Psi)$ is a cone and there exist constants $a_{0}, a_{1}, b_{0}, b_{1} \geq 0, \alpha>0$, and $\sigma \geq 1$ such that

$$
\begin{gathered}
\Psi(u)-\alpha \Psi^{\prime}(u ; u) \geq a_{0}\|u\|_{X}^{\sigma}-a_{1}, \quad \forall u \in D(\Psi), \\
\mathscr{F}(u)-\alpha\left(\mathscr{F}_{X}\right)^{0}(u ; u) \geq-b_{0}\|u\|_{X}^{\sigma}-b_{1}, \quad \forall u \in D(\Psi), \\
a_{0}>b_{0}+\alpha \quad \text { if } \sigma=1, \quad a_{0}>b_{0} \quad \text { if } \sigma>1 ;
\end{gathered}
$$

(H2) the following condition of $\left(S_{+}\right)$type is satisfied: if $\left\{u_{n}\right\}$ is a sequence in $D(\Psi)$ provided $u_{n} \rightarrow u$ weakly in $X$ and $\limsup _{n \rightarrow \infty}\left(-\Psi^{\prime}\left(u_{n} ; u-u_{n}\right)\right) \leq 0$, then $u_{n} \rightarrow u$ strongly in $X$.

Then the functional $\Phi$ satisfies the Palais-Smale condition in the sense of Definition 2.2.

Proof. Let $\left\{u_{n}\right\}$ be a sequence in $X$ for which there is a constant $M>0$ with

$$
\left|\Phi\left(u_{n}\right)\right| \leq M, \quad \forall n \geq 1
$$

and inequality (2.4) holds for $F=\mathscr{F}_{X}$ and a sequence $\varepsilon_{n} \rightarrow 0^{+}$. By (3.12), each $u_{n}$ is in $D(\Psi)$. For $t>0$, set $v=(1+t) u_{n}$ in (2.4) with $F=\mathscr{F}_{X}$. Dividing by $t$ and then letting $t \backslash 0$, one obtains that

$$
\Psi^{\prime}\left(u_{n} ; u_{n}\right)+\left(\mathscr{F}_{X}\right)^{0}\left(u_{n} ; u_{n}\right) \geq-\varepsilon_{n}\left\|u_{n}\right\|_{X}, \quad \forall n \geq 1
$$


Inequalities (3.12) and (3.13) ensure that for $n$ sufficiently large, one has

$$
\begin{aligned}
M+\alpha\left\|u_{n}\right\|_{X} & \geq \Psi\left(u_{n}\right)+\mathscr{F}\left(u_{n}\right)+\alpha \varepsilon_{n}\left\|u_{n}\right\|_{X} \\
& \geq \Psi\left(u_{n}\right)-\alpha \Psi^{\prime}\left(u_{n} ; u_{n}\right)+\left[\mathscr{F}\left(u_{n}\right)-\alpha\left(\left.\mathscr{F}\right|_{X}\right)^{0}\left(u_{n} ; u_{n}\right)\right] .
\end{aligned}
$$

Using (3.9) and (3.10), we find that

$$
M+\alpha\left\|u_{n}\right\|_{X} \geq\left(a_{0}-b_{0}\right)\left\|u_{n}\right\|_{X}^{\sigma}-a_{1}-b_{1} .
$$

Then (3.11) and (3.15) show that $\left\{u_{n}\right\}$ is bounded in $X$. By the compactness of the embedding of $X$ into $Z$, the sequence $\left\{u_{n}\right\}$ contains a subsequence, again denoted by $\left\{u_{n}\right\}$ such that

$$
\begin{array}{ll}
u_{n} \longrightarrow u & \text { weakly in } X, \\
u_{n} \longrightarrow u & \text { strongly in } Z,
\end{array}
$$

for some $u \in X$. Now put $v=u_{n}+t\left(u-u_{n}\right), t>0$, in (2.4) with $F=\left.\mathscr{F}\right|_{X}$. Similar to $(3.13)$, we derive that

$$
\Psi^{\prime}\left(u_{n} ; u-u_{n}\right)+\left(\mathscr{F}_{X}\right)^{0}\left(u_{n} ; u-u_{n}\right) \geq-\varepsilon_{n}\left\|u-u_{n}\right\|_{X}, \quad \forall n \geq 1 .
$$

This implies

$$
\Psi^{\prime}\left(u_{n} ; u-u_{n}\right)+\mathscr{F}^{0}\left(u_{n} ; u-u_{n}\right) \geq-\varepsilon_{n}\left\|u-u_{n}\right\|_{X}, \quad \forall n \geq 1 .
$$

As $\left\{u_{n}\right\}$ is bounded in $X$, we infer from (3.17) and the upper semicontinuity of $\mathscr{F}^{0}$ that

$$
\liminf _{n \rightarrow \infty} \Psi^{\prime}\left(u_{n} ; u-u_{n}\right) \geq 0
$$

Taking into account (3.16) and (3.20), assumption (H2) completes the proof.

Remark 3.6. If $\Psi^{\prime}(u ; \cdot)$ is homogeneous, for all $u \in D(\Psi)$, then (H2) becomes the usual form of the $\left(S_{+}\right)$condition: if $\left\{u_{n}\right\}$ is a sequence in $D(\Psi)$ provided $u_{n} \rightarrow u$ weakly in $X$ and $\limsup _{n \rightarrow \infty} \Psi^{\prime}\left(u_{n} ; u_{n}-u\right) \leq 0$, then $u_{n} \rightarrow u$ strongly in $X$.

We can now state the following result.

Theorem 3.7. Let $\Phi$ be defined in (3.4) and assume Lemma 3.5(H1) and (H2) together with the following hypotheses. 
(H3) There exists an element $\bar{u} \in D(\Psi)$ such that

$$
\begin{gathered}
a_{1}+b_{1} \leq\left(a_{0}-b_{0}\right)\|\bar{u}\|_{X}^{\sigma}, \\
\Phi(\bar{u})<0 .
\end{gathered}
$$

(H4) There exists a constant $\rho>0$ such that

$$
\inf _{\|v\|_{X}=\rho} \Phi(v)>\Phi(0)
$$

Then $\Phi$ has a nontrivial critical point $u \in X$. In particular, problem (3.1) has a nontrivial solution.

Proof. We apply Theorem 2.3(ii) to the functional $\Phi$ in (3.4). Lemma 3.5 guarantees that $\Phi$ satisfies the Palais-Smale condition. It remains to check that $\Phi$ verifies condition (2.5) with $\|e\|_{X}>\rho$. To this end, we prove that one can choose $e=t \bar{u}$ (with $\bar{u}$ entering (H3)) if $t>0$ is sufficiently large.

First, note that $\bar{u} \neq 0$. Indeed, from (3.9), (3.10), and (3.21), we have

$$
\Phi(\bar{u})-\alpha\left[\Psi^{\prime}(\bar{u} ; \bar{u})+\left(\left.\mathscr{F}\right|_{X}\right)^{0}(\bar{u} ; \bar{u})\right] \geq 0
$$

which leads to a contradiction with (3.22) if $\bar{u}=0$.

We observe that, due to the fact that $\bar{u} \in D(\Psi)$ and since $D(\Psi)$ is a cone, the convex function $s \mapsto \Psi(s \bar{u})$ is locally Lipschitz on $(0,+\infty)$. A straightforward computation shows that

$$
\begin{aligned}
\partial_{s}\left(s^{-1 / \alpha} \Phi(s \bar{u})\right)= & \partial_{s}\left(s^{-1 / \alpha} \Psi(s \bar{u})+\left.s^{-1 / \alpha} \mathscr{F}\right|_{X}(s \bar{u})\right) \\
\subset & -\frac{1}{\alpha} s^{-1 / \alpha-1} \Psi(s \bar{u})+s^{-1 / \alpha} \partial_{s}(\Psi(s \bar{u})) \\
& +\left(-\frac{1}{\alpha} s^{-1 / \alpha-1} \mathscr{F}(s \bar{u})+s^{-1 / \alpha}\left\langle\partial\left(\left.\mathscr{F}\right|_{X}\right)(s \bar{u}), \bar{u}\right\rangle\right), \quad \forall s>0,
\end{aligned}
$$

where the notation $\partial_{s}$ stands for the generalized gradient with respect to $s$. For an arbitrary $t>1$, Lebourg's mean value theorem yields some $\tau=\tau(t) \in(1, t)$ such that

$$
t^{-1 / \alpha} \Phi(t \bar{u})-\Phi(\bar{u})=\xi(t-1)
$$

where $\left.\xi \in \partial_{s}\left(s^{-1 / \alpha} \Phi(s \bar{u})\right)\right|_{s=\tau}$. This implies

$$
\begin{aligned}
t^{-1 / \alpha} \Phi(t \bar{u})-\Phi(\bar{u}) \in \frac{1}{\alpha}(t-1) \tau^{-1 / \alpha-1}[ & \left(\left.\alpha \tau \partial_{s}(\Psi(s \bar{u}))\right|_{s=\tau}-\Psi(\tau \bar{u})\right) \\
& \left.+\left(-\mathscr{F}(\tau \bar{u})+\alpha\left\langle\partial\left(\left.\mathscr{F}\right|_{X}\right)(\tau \bar{u}), \tau \bar{u}\right\rangle\right)\right] .
\end{aligned}
$$


Then, taking into account the convexity of $s \mapsto \Psi(s \bar{u})$, the regularity property of a convex function (see Clarke [5, pages 39-40]) and relations (3.9) and (3.10), we get that

$$
\begin{aligned}
& \Phi(t \bar{u}) \leq t^{1 / \alpha} \Phi(\bar{u})+\frac{1}{\alpha} t^{1 / \alpha}(t-1) \tau^{-1 / \alpha-1}[\left(\alpha \Psi^{\prime}(\tau \overline{\mathcal{u}} ; \tau \bar{u})-\Psi(\tau \bar{u})\right) \\
&\left.+\left(-\mathscr{F}(\tau \bar{u})+\alpha\left(\left.\mathscr{F}\right|_{X}\right)^{0}(\tau \bar{u} ; \tau \bar{u})\right)\right] \\
& \leq t^{1 / \alpha} \Phi(\bar{u})+\frac{1}{\alpha} t^{1 / \alpha}(t-1) \tau^{-1 / \alpha-1}\left[-\left(a_{0}-b_{0}\right) \tau^{\sigma}\|\bar{u}\|_{X}^{\sigma}+a_{1}+b_{1}\right], \quad \forall t>1 .
\end{aligned}
$$

By (3.21) and because $\tau>1$, we derive that

$$
\Phi(t \bar{u}) \leq t^{1 / \alpha} \Phi(\bar{u}), \quad \forall t>1
$$

Then (3.29) and assumption (3.22) imply

$$
\lim _{t \rightarrow+\infty} \Phi(t \bar{u})=-\infty
$$

Now, by means of (3.30), we can choose $\bar{t}>0$ sufficiently large to satisfy

$$
\bar{t}\|\bar{u}\|_{X}>\rho, \quad \Phi(\bar{t} \bar{u}) \leq \Phi(0),
$$

for $\rho>0$ entering (H4). If we compare (3.23) and (3.31), it is seen that the requirement in (2.5) is achieved for $e=\bar{t} \bar{u}$. Theorem 2.3(ii) assures that $\Phi$ in (3.4) has a nontrivial critical point $u \in X$. Furthermore, Remark 3.3 shows that $u$ is a (nontrivial) solution of problem (3.1). The proof of Theorem 3.7 is thus complete.

In the final part of this section, we are concerned with the case when

$$
\Psi=\Psi_{C}:=\varphi+I_{C}
$$

where $C$ is a nonempty, closed, and convex subset of $X, I_{C}$ denotes the indicator function of $C$, and $\varphi: X \rightarrow \mathbb{R}$ is a convex, Gâteaux differentiable functional. Note that $\Psi_{C}$ is convex, l.s.c., and proper and $D\left(\Psi_{C}\right)=C$. Therefore, the functional

$$
\Phi=\Psi_{C}+\mathscr{F}_{X}
$$

with $\mathscr{F}$ as at the beginning of this section, has the form required in (3.4).

Consider the following problem of variational-hemivariational inequality type:

Find $u \in C$ such that $\left(\left.\mathscr{F}\right|_{X}\right)^{0}(u ; v-u)+\langle d \varphi(u), v-u\rangle \geq 0, \quad \forall v \in C$. 
Remark 3.8. (i) Taking into account that, for $u \in C$,

$$
\Psi_{C}^{\prime}(u ; v)= \begin{cases}\langle d \varphi(u), v\rangle & \text { if } u+t v \in C \text { for some } t \in(0,1] \\ +\infty & \text { otherwise, }\end{cases}
$$

a straightforward computation shows that problem (3.34) is equivalent to the following problem of type (3.1):

Find $u \in D\left(\Psi_{C}\right)=C$ such that $\left(\mathscr{F}_{X}\right)^{0}(u ; v)+\Psi_{C}^{\prime}(u ; v) \geq 0, \quad \forall v \in X$.

(ii) If $C$ is a nonempty, closed, and convex cone, then each solution of problem (3.34) solves also the problem:

$$
\text { Find } u \in C \text { such that }\left(\left.\mathscr{F}\right|_{X}\right)^{0}(u ; v)+\langle d \varphi(u), v\rangle \geq 0, \quad \forall v \in C \text {. }
$$

Proposition 3.9. If $u \in X$ is a critical point of $\Phi$ in (3.33) and (3.32), then $u$ is a solution of problem (3.34).

Proof. Viewing Remark 3.8(i), the conclusion follows from Proposition 3.2(i).

Theorem 3.10. If the functional $\Phi$ in (3.33) and (3.32) is coercive on $X$, then problem (3.34) has a solution.

Proof. It is a direct consequence of Theorem 3.4 and Proposition 3.9.

Theorem 3.11. For the defining $\Phi$ data entering (3.33) and (3.32), we assume the following.

$\left(\mathrm{H} 1^{\prime}\right)$ The set $C$ is a nonempty, closed, and convex cone in $X$ and there exist constants $a_{0}, a_{1}, b_{0}, b_{1} \geq 0, \alpha>0$, and $\sigma \geq 1$ such that one has (3.11),

$$
\begin{gathered}
\varphi(u)-\alpha\langle d \varphi(u), u\rangle \geq a_{0}\|u\|_{X}^{\sigma}-a_{1}, \quad \forall u \in C, \\
\mathscr{F}(u)-\alpha\left(\left.\mathscr{F}\right|_{X}\right)^{0}(u ; u) \geq-b_{0}\|u\|_{X}^{\sigma}-b_{1}, \quad \forall u \in C .
\end{gathered}
$$

$\left(\mathrm{H} 2^{\prime}\right)$ The following condition of $\left(S_{+}\right)$type is satisfied: if $\left\{u_{n}\right\}$ is a sequence in $C$ provided $u_{n} \rightarrow u$ weakly in $X$ and $\limsup _{n \rightarrow \infty}\left\langle d \varphi\left(u_{n}\right), u_{n}-u\right\rangle \leq 0$, then $u_{n} \rightarrow u$ strongly in $X$.

(H3') There exists an element $\bar{u} \in C$ such that (3.21) holds with $a_{0}, a_{1}, b_{0}$, and $b_{1}$ from $\left(\mathrm{H}^{\prime}\right)$ together with

$$
\mathscr{F}(\bar{u})+\varphi(\bar{u})<0 .
$$

$\left(\mathrm{H} 4^{\prime}\right)$ There exists a constant $\rho>0$ such that

$$
\inf _{\substack{\|v\|=\rho \\ v \in C}}(\mathscr{F}(v)+\varphi(v))>\mathscr{F}(0)+\varphi(0) .
$$


Then $\Phi$ in (3.33) and (3.32) has a nontrivial critical point $u \in C$. In particular, problem (3.34) has a nontrivial solution.

Proof. Note that assumptions $\left(\mathrm{H}^{\prime}\right),\left(\mathrm{H} 2^{\prime}\right),\left(\mathrm{H} 3^{\prime}\right)$, and $\left(\mathrm{H} 4^{\prime}\right)$ are just $(\mathrm{H} 1),(\mathrm{H} 2)$, (H3), and (H4), respectively, in the case where $D(\Psi)=C$ is a closed convex cone and $\Psi$ is given by (3.32). Thus it suffices to apply Theorem 3.7 and Proposition 3.9 to the functional $\Phi$ in (3.33) and (3.32).

Remark 3.12. It is worth pointing out that if we take $C=X$, then problem (3.34) becomes

$$
\text { Find } u \in X \text {, such that } d \varphi(u) \in \partial\left(-\left.\mathscr{F}\right|_{X}\right)(u) \text {. }
$$

Thus, [8, Theorems 3.2 and 3.4] are immediate consequences of Theorems 3.10 and 3.11 , respectively.

\section{Applications to nonsmooth boundary value problems}

In order to illustrate how the abstract results of Section 3 can be applied, we consider a concrete problem of type (3.34). To this end, let $\Omega$ be a bounded domain in $\mathbb{R}^{N}, N \geq 1$, with Lipschitz-continuous boundary $\Gamma=\partial \Omega$ and let $\omega \subset$ $\bar{\Omega}$ be a measurable set. Given $p \in(1, \infty)$, the Sobolev space $W^{1, p}(\Omega)$ is endowed with its usual norm (see [1, page 44]).

We denote

$$
\begin{aligned}
& W_{0}=\left\{v \in W^{1, p}(\Omega):\left.v\right|_{\Gamma}=0\right\}, \\
& W_{1}=\left\{v \in W^{1, p}(\Omega): \int_{\Omega} v=0\right\}, \\
& W_{2}=\left\{v \in W_{1}:\left.v\right|_{\Gamma}=\text { constant }\right\} .
\end{aligned}
$$

In the sequel, $W$ will stand for any of the above (closed) subspaces $W_{0}, W_{1}$, and $W_{2}$ of $W^{1, p}(\Omega)$. By the Poincaré-Wirtinger inequality, the functional

$$
W \ni v \longmapsto\|v\|_{1, p}:=\left(\int_{\Omega}|\nabla v|^{p}\right)^{1 / p}
$$

is a norm on $W$, equivalent to the induced norm from $W^{1, p}(\Omega)$. The dual space $W^{*}$ is considered endowed with the dual norm of $\|\cdot\|_{1, p}$.

Now, we define the $p$-Laplacian operator $-\Delta_{p}: W \rightarrow W^{*}$ by

$$
\left\langle-\Delta_{p} u, v\right\rangle=\int_{\Omega}|\nabla u|^{p-2} \nabla u \nabla v, \quad \forall u, v \in W .
$$

Arguments similar to those in [7] show that the convex functional $\varphi: W \rightarrow \mathbb{R}$ defined by

$$
\varphi(u)=\frac{1}{p}\|u\|_{1, p}^{p}, \quad \forall u \in W
$$


is continuously differentiable on $W$ and its differential is $-\Delta_{p}$, that is,

$$
\langle d \varphi(u), v\rangle=\left\langle-\Delta_{p} u, v\right\rangle, \quad \forall u, v \in W
$$

Moreover, as $d \varphi$ is the duality mapping on $W$, corresponding to the gauge function $t \mapsto t^{p-1}$ and because $W$ is uniformly convex, $d \varphi$ satisfies condition $\left(S_{+}\right)$ (see Remark 3.6).

If $p^{*}$ stands for the Sobolev critical exponent, that is,

$$
p^{*}= \begin{cases}\frac{N p}{N-p} & \text { if } p<N \\ +\infty & \text { if } p \geq N\end{cases}
$$

then, for any fixed $q \in\left(1, p^{*}\right)$, by the Rellich-Kondrachov theorem, the embedding $W \hookrightarrow L^{q}(\Omega)$ is compact (the space $L^{q}(\Omega)$ is understood with its usual norm $\left.\|\cdot\|_{0, q}\right)$.

The results in Section 3 will be applied by taking $X=W, Z=L^{q}(\Omega)$, and $\varphi$ defined in (4.4).

Further, to complete the setting, let a function $g: \Omega \times \mathbb{R} \rightarrow \mathbb{R}$ be measurable and satisfy the growth condition

$$
|g(x, s)| \leq c_{1}|s|^{q-1}+c_{2} \quad \text { for a.e. } x \in \Omega, \forall s \in \mathbb{R},
$$

where $c_{1}, c_{2} \geq 0$ are constants. For a.e. $x \in \Omega$ and all $s \in \mathbb{R}$, we put

$$
\begin{aligned}
& \underline{g}(x, s)=\lim _{\delta \rightarrow 0^{+}} \underset{|t-s|<\delta}{\operatorname{essinf}} g(x, t), \\
& \bar{g}(x, s)=\lim _{\delta \rightarrow 0^{+}} \operatorname{ess\operatorname {sup}} g(x, t) .
\end{aligned}
$$

The following condition will be invoked below:

$$
\underline{g} \text { and } \bar{g} \text { are } N \text {-measurable }
$$

(recall that a function $h: \Omega \times \mathbb{R} \rightarrow \mathbb{R}$ is called $N$-measurable if $h(\cdot, u(\cdot)): \Omega \rightarrow \mathbb{R}$ is measurable whenever $u: \Omega \rightarrow \mathbb{R}$ is measurable).

By (4.7), the primitive $G: \Omega \times \mathbb{R} \rightarrow \mathbb{R}$ of function $g$ :

$$
G(x, s)=\int_{0}^{s} g(x, t) d t \quad \text { for a.e. } x \in \Omega, \forall s \in \mathbb{R}
$$

satisfies

$$
|G(x, s)| \leq \frac{c_{1}}{q}|s|^{q}+c_{2}|s| \quad \text { for a.e. } x \in \Omega, \forall s \in \mathbb{R} \text {. }
$$


612 Existence results for inequality problems

Taking into account (4.11), we define the functional $\mathscr{G}: L^{q}(\Omega) \rightarrow \mathbb{R}$ by putting

$$
\mathscr{G}(u)=-\int_{\Omega} G(x, u), \quad \forall u \in L^{q}(\Omega)
$$

It is known (see, e.g., Chang [4]) that $\mathscr{G}$ is Lipschitz continuous on the bounded subsets of $L^{q}(\Omega)$. At this stage, we introduce the closed convex cone $K$ in $W$ :

$$
K=\{u \in W: u(x) \geq 0 \text { for a.e. } x \in \omega\}
$$

and we formulate the problem:

Find $u \in K$ such that $\left(\left.\mathscr{G}\right|_{W}\right)^{0}(u ; v-u)+\left\langle-\Delta_{p} u, v-u\right\rangle \geq 0, \quad \forall v \in K$.

Thus, the functional framework in Section 3 is now accomplished by taking $\mathscr{F}=$ $\mathscr{G}$ and $C=K$. Clearly, problem (4.14) is of the same type as (3.34). Before passing on to obtaining existence results for problem (4.14), it should be noticed that the nonsmooth functional $\Phi=\Phi_{K}: W \rightarrow(-\infty,+\infty]$, defined by

$$
\Phi_{K}=\varphi_{W_{W}}+\varphi+I_{K}
$$

with $\varphi$ in (4.4), $I_{K}$ the indicator function of the cone $K$ in (4.13), has the form required in (3.33) and (3.32).

We also need to invoke the following constant, depending on the cone $K$ in the Banach space $W$ :

$$
\lambda_{1}=\lambda_{1, K}:=\inf \left\{\frac{\|v\|_{1, p}^{p}}{\|v\|_{0, p}^{p}}: v \in K \backslash\{0\}\right\} .
$$

Note that

$$
\|v\|_{0, p} \leq \lambda_{1}^{-1 / p}\|v\|_{1, p}, \quad \forall v \in K
$$

Theorem 4.1. Assume (4.7) together with

(i) $\limsup \sup _{s \rightarrow-\infty} p G(x, s) /|s|^{p}<\lambda_{1}$ uniformly for a.e. $x \in \Omega \backslash \omega$;

(ii) $\limsup _{s \rightarrow+\infty} p G(x, s) / s^{p}<\lambda_{1}$ uniformly for a.e. $x \in \Omega$.

Then problem (4.14) has a solution.

Proof. By Theorem 3.10, it suffices to show that the functional $\Phi_{K}$ in (4.15) is coercive on $W$.

From (i) and (ii), there are numbers $\varepsilon \in\left(0, \lambda_{1}\right)$ and $s_{0}>0$ such that

$$
\begin{aligned}
& G(x, s) \leq \frac{\lambda_{1}-\varepsilon}{p}|s|^{p} \quad \text { for a.e. } x \in \Omega \backslash \omega, \forall s<-s_{0}, \\
& G(x, s) \leq \frac{\lambda_{1}-\varepsilon}{p} s^{p} \quad \text { for a.e. } x \in \Omega, \forall s>s_{0} .
\end{aligned}
$$


Using (4.11), we can find a positive constant $k=k\left(s_{0}\right)$ with

$$
|G(x, s)| \leq k \quad \text { for a.e. } x \in \Omega, \forall s \in\left[-s_{0}, s_{0}\right] .
$$

For $u \in K$, we put

$$
\Omega_{-}:=\{x \in \Omega: u<0\}, \quad \Omega_{+}:=\Omega \backslash \Omega_{-} .
$$

Notice that by (4.13) we have $\Omega_{-} \subset \Omega \backslash \omega$. Then by (4.18) and (4.20), it follows that

$$
\begin{aligned}
\int_{\Omega_{-}} G(x, u) & =\int_{\left[u<-s_{0}\right]} G(x, u)+\int_{\left[-s_{0} \leq u<0\right]} G(x, u) \\
& \leq \frac{\lambda_{1}-\varepsilon}{p} \int_{\Omega_{-}}|u|^{p}+k|\Omega| .
\end{aligned}
$$

On the other hand, by (4.19) and (4.20), one sees that

$$
\begin{aligned}
\int_{\Omega_{+}} G(x, u) & =\int_{\left[u>s_{0}\right]} G(x, u)+\int_{\left[0 \leq u \leq s_{0}\right]} G(x, u) \\
& \leq \frac{\lambda_{1}-\varepsilon}{p} \int_{\Omega_{+}}|u|^{p}+k|\Omega| .
\end{aligned}
$$

Combining (4.22) and (4.23), the following estimate holds:

$$
\int_{\Omega} G(x, u) \leq 2 k|\Omega|+\frac{\lambda_{1}-\varepsilon}{p}\|u\|_{0, p}^{p}, \quad \forall u \in K .
$$

Then, from (4.15), it follows that

$$
\Phi_{K}(u)=\frac{1}{p}\|u\|_{1, p}^{p}-\int_{\Omega} G(x, u) \geq \frac{1}{p}\|u\|_{1, p}^{p}-\frac{\lambda_{1}-\varepsilon}{p}\|u\|_{0, p}^{p}-2 k|\Omega|, \quad \forall u \in W .
$$

By (4.17), we infer

$$
\Phi_{K}(u) \geq \frac{\varepsilon}{p \lambda_{1}}\|u\|_{1, p}^{p}-2 k|\Omega|, \quad \forall u \in W
$$

showing that

$$
\lim _{\|u\|_{1, p \rightarrow \infty}} \Phi_{K}(u)=+\infty
$$

Theorem 4.2. Assume (4.7), (4.9), and $\operatorname{int}(\Omega \backslash \omega) \neq \varnothing$ if $W=W_{1}$ or $W=W_{2}$, together with

(i) $\limsup _{s>0} p G(x, s) /|s|^{p}<\lambda_{1}$ uniformly for a.e. $x \in \Omega \backslash \omega$;

(ii) $\limsup _{s \backslash 0} p G(x, s) / s^{p}<\lambda_{1}$ uniformly for a.e. $x \in \Omega$; 
614 Existence results for inequality problems

and there are numbers $\theta>p$ and $s_{0}>0$ such that

(iii) $0<\theta G(x, s) \leq s g(x, s)$ for a.e. $x \in \Omega \backslash \omega, \forall s \leq-s_{0}$,

(iv) $0<\theta G(x, s) \leq s g(x, s)$ for a.e. $x \in \Omega, \forall s \geq s_{0}$.

Then problem (4.14) has a nontrivial solution.

Proof. We will apply Theorem 3.11. Without loss of generality, we may suppose in (4.7) that $q \in\left(p, p^{*}\right)$. For $u \in K$ (see (4.13)), the sets $\Omega_{-}$and $\Omega_{+}$will be considered as being defined by (4.21), and recall that $\Omega_{-} \subset \Omega \backslash \omega$.

First we check $\left(H 4^{\prime}\right)$. By (i) and (ii), one can find numbers $\varepsilon \in\left(0, \lambda_{1}\right)$ and $\delta_{0}>0$ such that

$$
\begin{array}{ll}
G(x, s) \leq \frac{\lambda_{1}-\varepsilon}{p}|s|^{p} \quad \text { for a.e. } x \in \Omega \backslash \omega, \forall s \in\left[-\delta_{0}, 0\right), \\
G(x, s) \leq \frac{\lambda_{1}-\varepsilon}{p}|s|^{p} \quad \text { for a.e. } x \in \Omega, \forall s \in\left(0, \delta_{0}\right] .
\end{array}
$$

From (4.11), there exists a constant $c=c\left(\delta_{0}\right)$ with

$$
G(x, s) \leq c|s|^{q} \quad \text { for a.e. } x \in \Omega, \forall|s|>\delta_{0} .
$$

For an arbitrary $u \in K$, by (4.28) and (4.30) we have

$$
\begin{aligned}
\int_{\Omega_{-}} G(x, u) & =\int_{\Omega_{-} \cap\left[-\delta_{0} \leq u\right]} G(x, u)+\int_{\left[u<-\delta_{0}\right]} G(x, u) \\
& \leq \frac{\lambda_{1}-\varepsilon}{p} \int_{\Omega_{-}}|u|^{p}+c \int_{\Omega_{-}}|u|^{q} .
\end{aligned}
$$

Similarly, (4.29) and (4.30) imply

$$
\begin{aligned}
\int_{\Omega_{+}} G(x, u) & =\int_{\Omega_{+} \cap\left[u \leq \delta_{0}\right]} G(x, u)+\int_{\left[u>\delta_{0}\right]} G(x, u) \\
& \leq \frac{\lambda_{1}-\varepsilon}{p} \int_{\Omega_{+}}|u|^{p}+c \int_{\Omega_{+}}|u|^{q} .
\end{aligned}
$$

Then, combining (4.31) and (4.32), we infer

$$
\int_{\Omega} G(x, u) \leq \frac{\lambda_{1}-\varepsilon}{p}\|u\|_{0, p}^{p}+c\|u\|_{0, q}^{q} .
$$

Taking into account the continuity of the embedding $W \hookrightarrow L^{q}(\Omega)$, from (4.33) and (4.17) we get, for a constant $\tilde{c}$, the relations

$$
\begin{aligned}
\mathscr{G}(u)+\varphi(u) & =-\int_{\Omega} G(x, u)+\frac{1}{p}\|u\|_{1, p}^{p} \geq \frac{\varepsilon}{\lambda_{1} p}\|u\|_{1, p}^{p}-\tilde{c}\|u\|_{1, p}^{q}>0 \\
& =\mathscr{G}(0)+\varphi(0)
\end{aligned}
$$


provided $u \in K$ and $\|u\|_{1, p}=\rho>0$ is sufficiently small. Therefore, Theorem $3.11\left(\mathrm{H} 4^{\prime}\right)$ is satisfied.

To check hypothesis $\left(\mathrm{H1}^{\prime}\right)$, we proceed as follows. From (iv), we have

$$
\frac{G(x, s)}{s} \leq \frac{1}{\theta} g(x, s) \quad \text { for a.e. } x \in \Omega, \forall s \geq s_{0} .
$$

For a.e. $x \in \Omega$, the primitive $G(x, s)$ as a function of $s$ being continuous (even locally Lipschitz), (4.35) implies

$$
\frac{G(x, s)}{s} \leq \frac{1}{\theta} \underline{g}(x, s) \quad \text { for a.e. } x \in \Omega, \forall s>s_{0} .
$$

Similarly, by (iii), we get

$$
G(x, s) \leq \frac{1}{\theta} s \bar{g}(x, s) \quad \text { for a.e. } x \in \Omega \backslash \omega, \forall s<-s_{0} .
$$

Recall that under the assumptions (4.7) and (4.9), for $u \in L^{q}(\Omega)$, the following inclusion holds (see [4, Theorem 2.1]):

$$
\partial(-\mathscr{G})(u) \subset[\underline{g}(x, u), \bar{g}(x, u)] \quad \text { for a.e. } x \in \Omega \text {. }
$$

Then, from (4.20), (4.36), (4.37), (4.38), and (4.7), for an arbitrary $u \in K$, we obtain

$$
\begin{aligned}
-\mathscr{G}(u) & =\int_{\Omega} G(x, u)=\int_{\left[u<-s_{0}\right]} G(x, u)+\int_{\left[u>s_{0}\right]} G(x, u)+\int_{\left[-s_{0} \leq u \leq s_{0}\right]} G(x, u) \\
& \leq \frac{1}{\theta}\left[\int_{\left[u<-s_{0}\right]} u \bar{g}(x, u)+\int_{\left[u>s_{0}\right]} u \underline{g}(x, u)\right]+k|\Omega| \\
& \leq \frac{1}{\theta}\left[\int_{\left[u<-s_{0}\right]} u w+\int_{\left[u>s_{0}\right]} u w\right]+k|\Omega| \\
& =\frac{1}{\theta}\left[\int_{\Omega} u w-\int_{\left[|u| \leq s_{0}\right]} u w\right]+k|\Omega| \\
& \leq \frac{1}{\theta} \int_{\Omega} u w+k_{0}, \quad \forall w \in \partial(-\mathscr{G})(u),
\end{aligned}
$$

for a constant $k_{0}>0$. As $\partial(-\mathscr{G})(u)=-\partial \mathscr{G}(u)$, it follows that

$$
\mathscr{G}(u) \geq \frac{1}{\theta} \int_{\Omega} u w-k_{0}, \quad \forall w \in \partial \mathscr{G}(u)
$$


616 Existence results for inequality problems

Taking the supremum over $w \in \partial \mathscr{G}(u)$ in $(4.40)$, we deduce

$$
\mathscr{G}(u)-\frac{1}{\theta}\left(\left.\mathscr{G}\right|_{W}\right)^{0}(u ; u) \geq-k_{0}, \quad \forall u \in K .
$$

By virtue of (4.4) and (4.5), one has

$$
\varphi(u)-\frac{1}{\theta}\langle d \varphi(u), u\rangle=\left(\frac{1}{p}-\frac{1}{\theta}\right)\|u\|_{1, p}^{p}, \quad \forall u \in W .
$$

From (4.41) and (4.42), it turns out that Theorem 3.11(H1') is fulfilled with

$$
\alpha=\frac{1}{\theta}, \quad a_{0}=\frac{1}{p}-\frac{1}{\theta}, \quad a_{1}=0, \quad \sigma=p, \quad b_{0}=0, \quad b_{1}=k_{0} .
$$

To check condition Theorem 3.11(H3'), we first note that, on the basis of (i), (ii) and arguing as in the proof of [7, Proposition 7], one has

$$
\begin{aligned}
& G(x, t) \geq \gamma_{1}(x) t^{\theta} \quad \text { for a.e. } x \in \Omega, \forall t>s_{0}, \\
& G(x, t) \geq \gamma_{2}(x)|t|^{\theta} \quad \text { for a.e. } x \in \Omega \backslash \omega, \forall t<-s_{0},
\end{aligned}
$$

where $\gamma_{1}, \gamma_{2} \in L^{\infty}(\Omega), \gamma_{1}(x)>0$ for a.e. $x \in \Omega$, and $\gamma_{2}(x)>0$ for a.e. $x \in \Omega \backslash \omega$. Since, by assumption, $\operatorname{int}(\Omega \backslash \omega) \neq \varnothing$ if $W=W_{1}$ or $W=W_{2}$, there is some $\bar{u} \in$ $K$ such that $|\Omega(\bar{u})|>0$, where $\Omega(\bar{u})=\left\{x \in \Omega: \bar{u}>s_{0}\right\}$. For $t \geq 1$, using (4.20), (4.44), (4.45), and the inclusion $\left[t \bar{u}<-s_{0}\right] \subset \Omega \backslash \omega$, we estimate $-\mathscr{G}$ as follows:

$$
\begin{aligned}
-\mathscr{G}(t \bar{u}) & =\int_{\left[t|\bar{u}|>s_{0}\right]} G(x, t \bar{u})+\int_{\left[t|\bar{u}| \leq s_{0}\right]} G(x, t \bar{u}) \\
& \geq \int_{\left[t|\bar{u}|>s_{0}\right]} G(x, t \bar{u})-k|\Omega| \\
& =\int_{\left[t \bar{u}>s_{0}\right]} G(x, t \bar{u})+\int_{\left[t \bar{u}<-s_{0}\right]} G(x, t \bar{u})-k|\Omega| \\
& \geq t^{\theta}\left[\int_{\Omega(\bar{u})} \gamma_{1}(x) \bar{u}^{\theta}+\int_{\left[t \bar{u}<-s_{0}\right]} \gamma_{2}(x)|\bar{u}|^{\theta}\right]-k|\Omega| \\
& \geq t^{\theta} \int_{\Omega(\bar{u})} \gamma_{1}(x) \bar{u}^{\theta}-k|\Omega| .
\end{aligned}
$$

Therefore,

$$
\mathscr{G}(t \bar{u})+\varphi(t \bar{u}) \leq-t^{\theta} \int_{\Omega(\bar{u})} \gamma_{1}(x) \bar{u}^{\theta}+\frac{t^{p}}{p}\|\bar{u}\|_{1, p}^{p}+k|\Omega|, \quad \forall t \geq 1 .
$$

Taking into account $\theta>p$, it follows that $\Phi_{K}(t \bar{u}) \rightarrow-\infty$ as $t \rightarrow+\infty$. This establishes $\left(\mathrm{H} 3^{\prime}\right)$ with $\bar{u}$ replaced by $t \bar{u}$, for some $t \geq 1$ sufficiently large. 
Finally, hypothesis $\left(\mathrm{H}^{\prime}\right)$ is also satisfied because, as we have already noted, the duality mapping $d \varphi$ verifies condition $\left(S_{+}\right)$.

The application of Theorem 3.11 concludes the proof.

Remark 4.3. If $\omega=\varnothing$, then $K=W$. Taking into account Remark 3.12, in this case, problem (4.14) becomes

$$
\text { Find } u \in W \text { such that }-\Delta_{p} u \in \partial\left(-\mathscr{G}_{\mid}\right)(u) \text {. }
$$

This means that for $u \in W$, it corresponds $h \in \partial\left(-\left.\mathscr{G}\right|_{W}\right)(u) \subset \partial(-\mathscr{G})(u) \subset$ $L^{q^{\prime}}(\Omega)$, with $1 / q+1 / q^{\prime}=1$, such that $u$ satisfies the variational equality

$$
\int_{\Omega}\left(|\nabla u|^{p-2} \nabla u \nabla v+h v\right)=0, \quad \forall v \in W .
$$

Assuming (4.7) and (4.9), inclusion (4.38) and equality (4.49) show that each solution of problem (4.48) for $W=W_{0}$ also solves the differential inclusion problem:

Find $u \in W_{0}=W_{0}^{1, p}(\Omega)$ such that $-\Delta_{p} u \in[\underline{g}(x, u), \bar{g}(x, u)] \quad$ for a.e. $x \in \Omega$.

In the case $W=W_{1}$, denoting by $\widehat{w}=(1 /|\Omega|) \int_{\Omega} w$ the mean value of any $w \in$ $L^{1}(\Omega)$, relation (4.49) is expressed as follows:

$$
\int_{\Omega}\left(|\nabla u|^{p-2} \nabla u \nabla w+h(w-\widehat{w})\right)=0, \quad \forall w \in W^{1, p}(\Omega),
$$

or, equivalently,

$$
\int_{\Omega}\left[|\nabla u|^{p-2} \nabla u \nabla w+(h-\hat{h}) w\right)=0, \quad \forall w \in W^{1, p}(\Omega) .
$$

Thus, if $W=W_{1}$, with $u \in W$ in (4.48), the following problem is solved:

$$
\begin{aligned}
& \text { Find } u \in W_{1} \text { such that }-\Delta_{p} u \in[\underline{g}(x, u)-\widehat{\bar{g}(\cdot, u)}, \bar{g}(x, u) \\
& -\widehat{g(\cdot, u)}] \text { for a.e. } x \in \Omega \text {. }
\end{aligned}
$$

A problem similar to (4.53) is solved when $W=W_{2}$ in (4.48).

Corollary 4.4 (see [8, Theorem 5.1]). Assume (4.7), (4.9), and

$$
\limsup _{|s| \rightarrow+\infty} \frac{p G(x, s)}{|s|^{p}}<\lambda_{1, W_{0}} \quad \text { uniformly for a.e. } x \in \Omega \text {. }
$$

Then problem (4.50) has a solution. 
618 Existence results for inequality problems

Proof. Theorem 4.1 applies with $\omega=\varnothing$.

Corollary 4.5 (see [6, Theorem 3.6] and [8, Theorem 5.2]). Assume (4.7) and (4.9) together with

$$
\limsup _{s \rightarrow 0} \frac{p G(x, s)}{|s|^{p}}<\lambda_{1, W_{0}} \quad \text { uniformly for a.e. } x \in \Omega \text {. }
$$

If there are numbers $\theta>p$ and $s_{0}>0$ such that

$$
0<\theta G(x, s) \leq \operatorname{sg}(x, s) \quad \text { for a.e. } x \in \Omega, \forall|s| \geq s_{0},
$$

then problem (4.50) has a nontrivial solution.

Proof. We apply Theorem 4.2 with $\omega=\varnothing$.

\section{Acknowledgment}

The authors are grateful to the referee for valuable comments and suggestions.

\section{References}

[1] R. A. Adams, Sobolev Spaces, Pure and Applied Mathematics, vol. 65, Academic Press, New York, 1975.

[2] A. Ambrosetti and P. H. Rabinowitz, Dual variational methods in critical point theory and applications, J. Functional Analysis 14 (1973), 349-381.

[3] V. Barbu and Th. Precupanu, Convexity and Optimization in Banach Spaces, Mathematics and Its Applications (East European Series), vol. 10, D. Reidel, Dordrecht, 1986.

[4] K. C. Chang, Variational methods for nondifferentiable functionals and their applications to partial differential equations, J. Math. Anal. Appl. 80 (1981), no. 1, 102129.

[5] F. H. Clarke, Optimization and Nonsmooth Analysis, Canadian Mathematical Society Series of Monographs and Advanced Texts, John Wiley \& Sons, New York, 1983.

[6] G. Dinca, P. Jebelean, and J. Mawhin, A result of Ambrosetti-Rabinowitz type for pLaplacian, Qualitative Problems for Differential Equations and Control Theory, World Scientific Publishing, New Jersey, 1995, pp. 231-242.

[7] - Variational and topological methods for Dirichlet problems with p-Laplacian, Port. Math. (N.S.) 58 (2001), no. 3, 339-378.

[8] G. Dinca, P. Jebelean, and D. Motreanu, Existence and approximation for a general class of differential inclusions, Houston J. Math. 28 (2002), no. 1, 193-215.

[9] D. Motreanu and P. D. Panagiotopoulos, Minimax Theorems and Qualitative Properties of the Solutions of Hemivariational Inequalities, Nonconvex Optimization and Its Applications, vol. 29, Kluwer Academic Publishers, Dordrecht, 1999.

[10] P. H. Rabinowitz, Minimax Methods in Critical Point Theory with Applications to Differential Equations, CBMS Regional Conference Series in Mathematics, vol. 65, American Mathematical Society, District of Columbia, 1986. 
[11] A. Szulkin, Minimax principles for lower semicontinuous functions and applications to nonlinear boundary value problems, Ann. Inst. H. Poincaré Anal. Non Linéaire 3 (1986), no. 2, 77-109.

George Dincă: Department of Mathematics, University of Bucharest, St. Academiei, no. 14, 70109 Bucharest, Romania

E-mail address: dinca@cnfis.ro

Petru Jebelean: Department of Mathematics, West University of Timişoara, Bv. V. Pârvan, no. 4, 1900 Timişoara, Romania

E-mail address: jebelean@hilbert.math.uvt.ro

Dumitru Motreanu: Département de Mathématiques, Université de Perpignan, 52, avenue de Villeneuve, 66860 Perpignan Cedex, France

E-mail address: motreanu@univ-perp.fr 


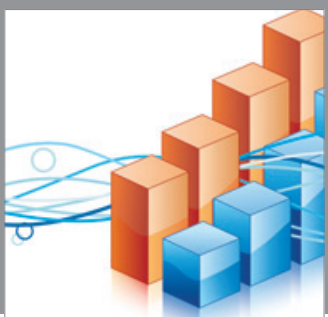

Advances in

Operations Research

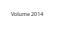

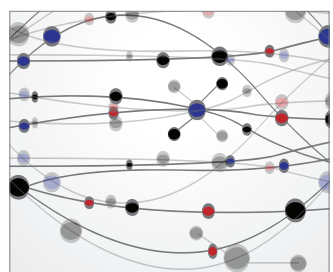

\section{The Scientific} World Journal
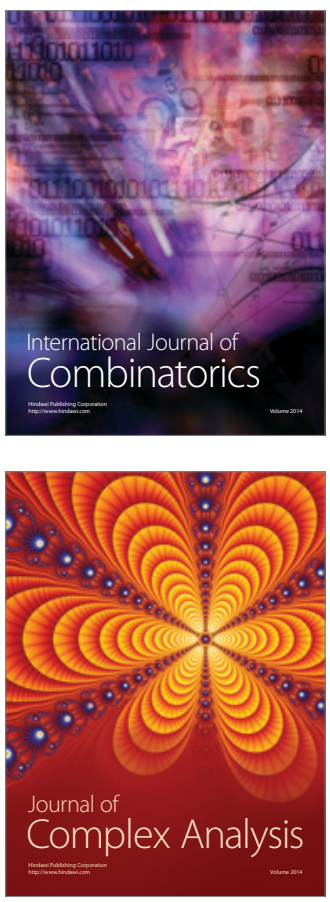

International Journal of

Mathematics and

Mathematical

Sciences
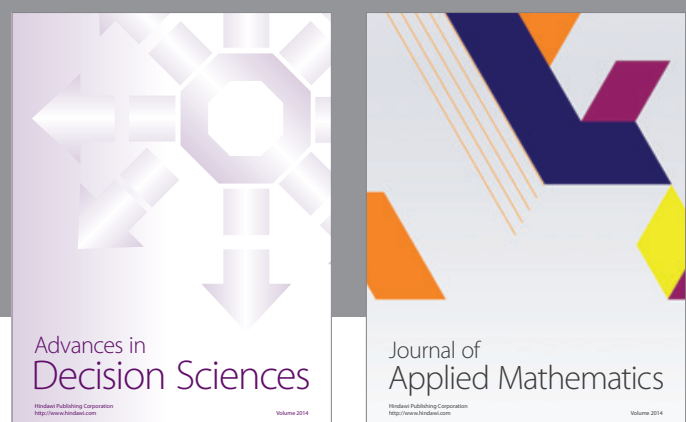

Journal of

Applied Mathematics
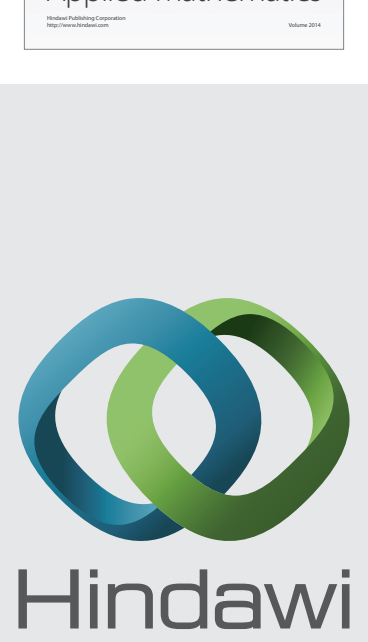

Submit your manuscripts at http://www.hindawi.com
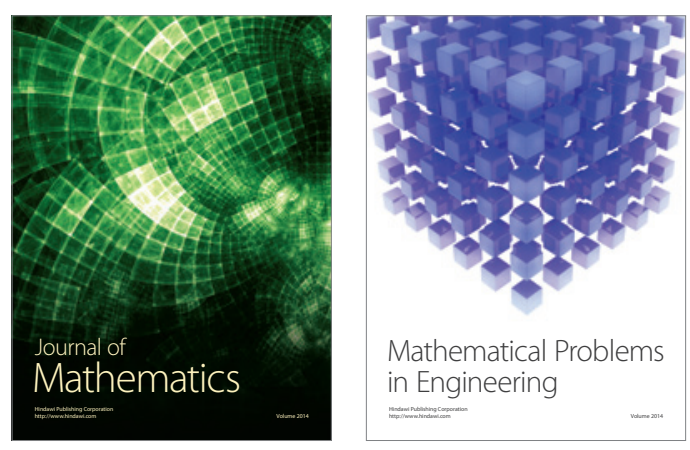

Mathematical Problems in Engineering
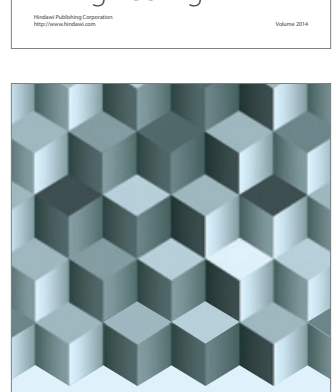

Journal of

Function Spaces
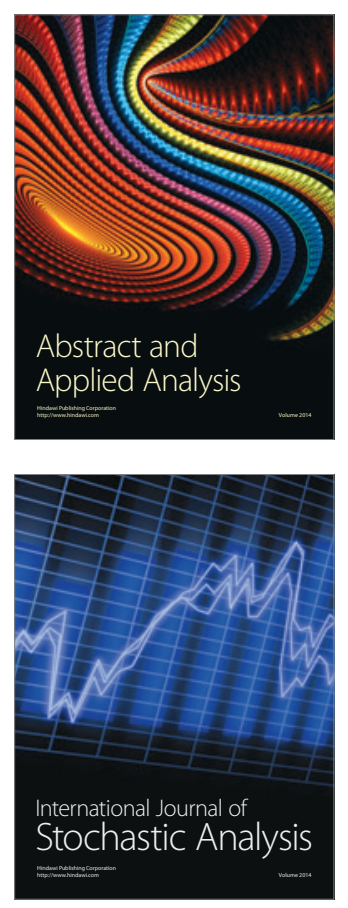

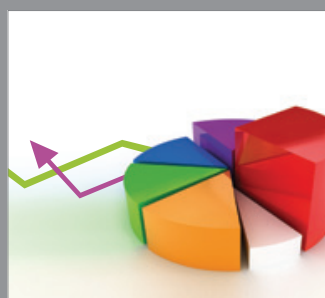

ournal of

Probability and Statistics

Promensencen
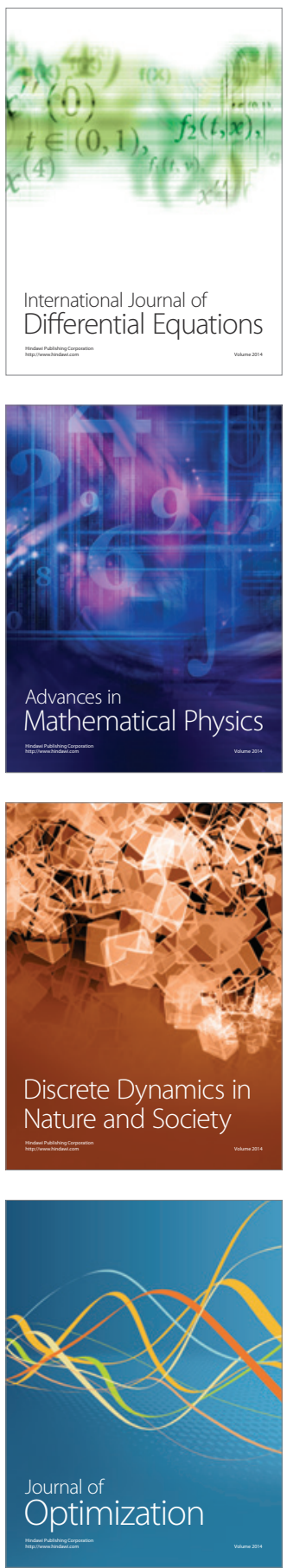